\title{
ON THE FINE STRUCTURE OF THE POLYGROUP BLOW-UP
}

\author{
ITAY BEN-YAACOV
}

\begin{abstract}
We study in detail the blow-up procedure described in [BTW01]. We obtain a structure theorem for coreless polygroups as a double quotient space $G / / H$, and a polygroup chunk theorem.

Seeking to remove the arbitrary parameter needed for the blow-up, we find canonical $\varnothing$-invariant groupoids $\mathcal{G}>\mathcal{H}$ analogous to $G$ and $H$ above, and show that $\mathcal{H}$ contains precisely all the arbitrary choices related to the blow-up.
\end{abstract}

\section{INTRODUCTION}

This paper continues [BTW01], seeking a better understanding of two fundamental concepts introduced therein: polygroups and the blow-up procedure.

In the discussions that led to the writing of [BTW01], one of the main sources of intuition was the double coset polygroup $G / / H=\{H a H: a \in G\}$. But then, this could be considered as a problem on its own: can any polygroup be written in this form? This is related to a conjecture made by Ivan Tomašić: that in a simple theory, any hyperdefinable polygroup is poly-isogenous to a group (two polygroups $P$ and $Q$ are poly-isogenous if there is a sub-polygroup of $P \times Q$ such that each projection has bounded fibres and an image of bounded index). For example, we know that if $H$ is commensurable with all its conjugates, then $G / / H$ is poly-isogenous to $G / N$ for some $N \triangleleft G$. He gave the following proof, using the yet-unproved (at the time) group configuration theorem: take three independent generic elements, obtain an algebraic quadrangle, then a group, then show the poly-isogeny.

Later on, in [BTW01], we defined the core of a polygroup and proved the following two properties, that allow a more direct and comprehensive approach:

- The set of generic elements in a gradedly almost hyperdefinable polygroup is type-definable. Thus we may obtain a polygroup chunk directly, without passing first through an algebraic quadrangle, a procedure by which we may well "lose" a part of the polygroup.

- [BTW01, Theorem 4.4], which gives a direct manner to obtain a map from the group to a coreless polygroup.

As we are looking for a poly-isogeny, it should be allowed to divide by a bounded normal sub-polygroup such as the core, and in fact it would seem to be necessary. So we allow ourselves to assume that the polygroup is coreless. In this case, the results mentioned above get us only as far as a surjective map $\hat{\pi}: G \rightarrow P$ such that $H=\operatorname{ker} \hat{\pi}$ is bounded, whence the poly-isogeny.

2000 Mathematics Subject Classification. 03C45,03C60.

Key words and phrases. simple theories - group configuration - polygroups.

The author would like to thank Frank O. Wagner for a stimulating discussion. 
In order to obtain a better result, a finer understanding of the kernel $H$ is required. We show that it is in fact intrinsic to the blow-up procedure (that is to say that it can be defined directly from the polygroup chunk: no polygroup $P$ or map $\hat{\pi}$ are required), and obtain some insight into its structure. As a corollary, we prove a stronger form of the conjecture: $P \simeq G / / H$. A Weil-Hrushovski coreless polygroup chunk theorem follows as well.

These are the principle results of this paper, and appear in the first section.

In the second section we derive certain structures from the blow-up procedure, with the following motivation in mind: since the polygroup chunk we started with is definable over $\varnothing$, we want to obtain a group definable over $\varnothing$ as well; or, if this is not possible, to describe by a $\varnothing$-definable structure the inevitable arbitrary choices that make this so. We give several descriptions of these arbitrary choices, the most elegant of which being given at the cost of the introduction of almost hyperdefinable groupoids.

It may seem that this question is completely unrelated to the first: however, we prove that the subgroup $H$ is, in a sense, precisely the set of such arbitrary choices. Moreover, originally it was this result that led to the understanding of the structure of $H$, and thence to the theorems of the first section, so the picture would be incomplete without it.

\section{Structure theOREM FOR CORELESS POLYGROUPS}

1.1. preliminaries. We recall from [Com84]:

Definition 1.1. A polygroup is a structure $\left\langle P, \cdot{ }^{-1}, e\right\rangle$ where ${ }^{-1}: P \rightarrow P$ is a map, $e \in P$ is a constant, and $\cdot$ is a multi-operation (namely $a \cdot b \subseteq P$ is a non-empty set for every $a, b \in P)$, such that:

1. For every $a, b, c \in P: a \cdot(b \cdot c)=(a \cdot b) \cdot c$ (as sets).

2. For every $a \in P: a \cdot e=e \cdot a=\{a\}$.

3. For every $a, b, c \in P: a \in b \cdot c \leftrightarrow b \in a \cdot c^{-1} \leftrightarrow c \in b^{-1} \cdot a$.

For general simplicity theory we refer the reader to [Wag00]. The reader should consult [BTW01] for many of the results and definitions we use in this paper, and in particular for the theory of almost hyperdefinable structures, almost hyperimaginaries and their theory of independence.

Definition 1.2. An polygroup is almost hyperdefinable in a theory $T$ if $\left\langle P, \cdot,{ }^{-1}, e\right\rangle$ is an almost hyperdefinable multi-structure (in T) in the sense of [BTW01]. In particular, the set $a \cdot b$ is bounded for every $a, b \in P$.

Remark 1.3. For our needs, there is no need to assume that ${ }^{-1}$ is single-valued, nor that e exists: this follows anyways from corelessness (see below) which is why these assumption are omitted in [BTW01].

When $T$ is simple we also have the notion of a polygroup chunk:

Definition 1.4. (T simple)

An almost hyperdefinable generic polygroup chunk $\left\langle S, \cdot,{ }^{-1}\right\rangle$ is defined similarly to a polygroup: 
1. If $a, b \in S$ are independent then $a \cdot b \neq \varnothing$ (otherwise it may be empty), and then $c \downarrow a$ and $c \downarrow b$ for every $c \in a \cdot b$.

2. For independent $a, b, c \in S: a \cdot(b \cdot c)=(a \cdot b) \cdot c$ (as sets).

3. For pairwise independent $a, b, c \in S: a \in b \cdot c \leftrightarrow b \in a \cdot c^{-1} \leftrightarrow c \in b^{-1} \cdot a$.

Convention 1.5. $T$ is a simple theory.

By definable we mean almost hyperdefinable (in T), and by a polygroup (chunk) we mean an almost hyperdefinable polygroup (chunk). Unless explicitly said otherwise, all are defined over $\varnothing$.

Generic elements are defined for polygroups as they are defined for groups, and we prove in [BTW01] that almost hyperdefinable polygroups in simple theories have generic elements, and the set of the generic elements of such a polygroup is a polygroup chunk.

Definition 1.6. 1. A polygroup $P$ is said to be coreless if whenever $a, b, g \in P$ such that $g$ is generic, $g \downarrow a, b$ and $a \cdot g \cap b \cdot g \neq \varnothing$ then $a=b$.

2. A polygroup chunk $S$ is said to be coreless if whenever $a, b, g \in S$, such that $g \downarrow a, b$ and $a \cdot g \cap b \cdot g \neq \varnothing$ then $a=b$.

In particular, the set of generic elements of a coreless polygroup is a coreless polygroup chunk. If $P$ is any polygroup, then there is a minimal normal sub-polygroup $N \triangleleft P$ such that $P / N$ is coreless, and moreover $N$ is bounded. We call $N$ the core of $P$.

We recall [BTW01, Lemma 3.2]:

Fact 1.7. If $S$ is a coreless polygroup chunk, and $a_{1}, a_{2}, b_{1}, b_{2} \in S$ are such that $a_{1}^{-1}$. $b_{1} \cap a_{2} \cdot b_{2}^{-1} \neq \varnothing$ and $\left\{a_{1}, b_{1}, b_{2}\right\}$ are independent, then $a_{1} \cdot a_{2} \cap b_{1} \cdot b_{2}=\{f\}$ is $a$ singleton.

Notation 1.8. In this case we write $f=a_{1} \cdot a_{2} \stackrel{c}{\cap} b_{1} \cdot b_{2}$ to say that $a_{1} \cdot a_{2} \cap b_{1} \cdot b_{2}$ is the singleton $\{f\}$, where $c \in a_{1}^{-1} \cdot b_{1} \cap a_{2} \cdot b_{2}^{-1}$ witnesses that that $a_{1} \cdot a_{2} \cap b_{1} \cdot b_{2} \neq \varnothing$.

Remark 1.9. The independence of the triplet $\left\{a_{1}, b_{1}, b_{2}\right\}$ is equivalent to the independence of any triplet from $\left\{a_{1}, a_{2}, b_{1}, b_{2}, c\right\}$, except for $\left\{a_{1}, a_{2}, c\right\}$ and $\left\{b_{1}, b_{2}, c\right\}$.

We now have everything we need in order to define the blow-up:

Let $S$ be a coreless polygroup chunk, and write $S=S_{0} / R$, meaning that $S_{0}$ is a typedefinable set and $R$ is the graded almost type-definable equivalence relation modulo which $S$ is defined. Fix some element $e \in S_{0}$; we are going to use $e$ as a parameter and work over it, so we need it to be at least hyperimaginary.

We define $\tilde{S}_{0}=\left\{\left(a, a^{\prime}, a^{\prime \prime}\right): a \in S_{0}, a \downarrow e, a^{\prime} \in e^{-1} \cdot a\right.$ and $\left.a^{\prime \prime} \in a \cdot e\right\} \subseteq S_{0}^{3}$, and $\tilde{S}=\tilde{S}_{0} / R \subseteq S^{3}$ (to be precise, $\tilde{S}=\tilde{S}_{0} /(R \times R \times R)$ ). A triplet $\left(a, a^{\prime}, a^{\prime \prime}\right) \in \tilde{S}$ is (ambiguously) denoted by $\tilde{a}$ : $a$ is its axis, and $a^{\prime}$ and $a^{\prime \prime}$ are the left-hand and righthand extensions, respectively. We also define the blow-up map $\pi: \tilde{S} \rightarrow S$ by $\pi(\tilde{a})=a$.

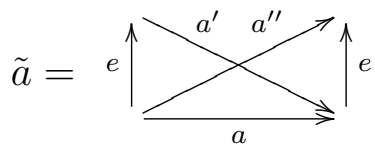


Assume that $\tilde{a}=\left(a, a^{\prime}, a^{\prime \prime}\right), \tilde{b}=\left(b, b^{\prime}, b^{\prime \prime}\right) \in \tilde{S}$, and $\tilde{a} \downarrow_{e} \tilde{b}$ (equivalently: $a \downarrow_{e} b$, or $a, b, e$ are independent). Then we can define $c=a \cdot b \stackrel{e^{\circ}}{\cap} a^{\prime \prime} \cdot b^{\prime}, c^{\prime}=e^{-1} \cdot c \stackrel{a}{\cap} a^{\prime} \cdot b$, $c^{\prime \prime}=a \cdot b^{\prime \prime} \stackrel{b}{\cap} c \cdot e$ and $\tilde{c}=\left(c, c^{\prime}, c^{\prime \prime}\right) \in \tilde{S}$.

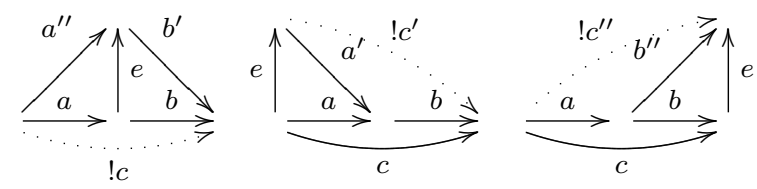

Define in this case $\tilde{a} \cdot \tilde{b}=\tilde{c}$ : then it is proved in [BTW01] that with this product (and $\left.\left(a, a^{\prime}, a^{\prime \prime}\right)^{-1}=\left(a^{-1}, a^{\prime \prime-1}, a^{\prime-1}\right)\right), \tilde{S}$ is an almost hyperdefinable group chunk (over the parameter $e$ ), so there is a unique group $G$, almost hyperdefinable over $e$, whose set of generic elements is isomorphic to $\tilde{S}$.

Convention 1.10. For simplicity, we add e to the language for the rest of this section, so independencies are over $e$, and every element of $S$ we consider (except for $e_{R}$ itself) is independent of $e$.

We identify the generic part of $G$ with $\tilde{S}$.

1.2. The subgroup $H$. We start with a technical lemma:

Lemma 1.11. Let $\tilde{a}=\left(a, a^{\prime}, a^{\prime \prime}\right)$ and $\tilde{b}_{i}=\left(b, b_{i}^{\prime}, b^{\prime \prime}\right)$ for $i<2$ be in $\tilde{S}$ such that $a \downarrow b$. Let $c=a \cdot b \stackrel{e}{\cap} a^{\prime \prime} \cdot b_{0}^{\prime}$ and $d=a \cdot e \stackrel{b}{\cap} c \cdot b_{1}^{\prime-1}$. Then $\left(\tilde{a} \cdot \tilde{b}_{0}\right) \cdot \tilde{b}_{1}^{-1}=\left(a, a^{\prime}, d\right)$.

Similarly, on the left.

Proof. Clearly $a \downarrow c$. Take $\tilde{c}=\left(c, c^{\prime}, c^{\prime \prime}\right)$ such that:

$$
\begin{aligned}
c & =a \cdot b \stackrel{e}{\cap} a^{\prime \prime} \cdot b_{0}^{\prime} \\
c^{\prime} & =e^{-1} \cdot c \stackrel{a}{\cap} a^{\prime} \cdot b \\
c^{\prime \prime} & =a \cdot b^{\prime \prime} \stackrel{b}{\cap} c \cdot e
\end{aligned} \quad \Longrightarrow \quad \tilde{c}=\tilde{a} \cdot \tilde{b}_{0}
$$

And then:

$$
\begin{aligned}
& a=c \cdot b^{-1} \stackrel{e}{\cap} c^{\prime \prime} \cdot b^{\prime \prime-1} \\
& a^{\prime}=e^{-1} \cdot a \stackrel{c}{\cap} c^{\prime} \cdot b^{-1} \\
& d=a \cdot e \stackrel{b}{\cap} c \cdot b_{1}^{\prime-1}
\end{aligned} \quad \Longrightarrow \quad \tilde{c} \cdot \tilde{b}_{1}^{-1}=\left(a, a^{\prime}, d\right)
$$

Definition 1.12. We define $H \subseteq G$ as the set of all $\left(a, a_{0}^{\prime}, a^{\prime \prime}\right) \cdot\left(a, a_{1}^{\prime}, a^{\prime \prime}\right)^{-1}$ for $a \in S$, $a_{i}^{\prime} \in e^{-1} \cdot a, a^{\prime \prime} \in a \cdot e$.

Proposition 1.13. Let $\tilde{a}=\left(a, a^{\prime}, a^{\prime \prime}\right) \in \tilde{S}$. Then there is a bijection $f: H \rightarrow a \cdot e$, given by $\tilde{a} \cdot \hat{b}=\left(a, a^{\prime}, f(\hat{b})\right)$ for every $\hat{b} \in H$, and this bijection does not depend on $a^{\prime}$. In other words, multiplying on the right by an element of $H$ has the sole effect of modifying the right-hand extension, every such modification is possible, and all this 
independently of the left-hand extension.

In yet other words, $\left(a_{0}, a_{0}^{\prime}, a_{0}^{\prime \prime}\right) \cdot\left(a_{1}, a_{1}^{\prime}, a_{1}^{\prime \prime}\right)^{-1} \in H$ if and only if $a_{0}=a_{1}=a$ and $a_{0}^{\prime \prime}=a_{1}^{\prime \prime}=a^{\prime \prime}$, and in this case the value of the product does not depend on the choice of $a^{\prime \prime} \in a \cdot e$, but only on $a, a_{0}^{\prime}$ and $a_{1}^{\prime}$.

Similarly for multiplying on the left.

Proof. We wish to calculate $\tilde{a} \cdot \hat{b}$ for $\hat{b} \in H$. Write $\hat{b}=\tilde{b}_{0} \cdot \tilde{b}_{1}^{-1}$ where $\tilde{b}_{i}=\left(b, b_{i}^{\prime}, b^{\prime \prime}\right)$. Take any $\tilde{c}_{0} \downarrow a b$, and let $\tilde{c}_{1}=\tilde{c}_{0} \cdot \hat{b}$. Then by Lemma 1.11 we can write $\tilde{c}_{i}=\left(c, c^{\prime}, c_{i}^{\prime \prime}\right)$ for $i<2$, and $\hat{b}=\tilde{c}_{0}^{-1} \cdot \tilde{c}_{1}$. But then again by Lemma 1.11 we have $\tilde{a} \cdot \hat{b}=\left(a, a^{\prime}, d\right)$ for some $d$, so $f(\hat{b})=d$ is well defined. Finally, Lemma 1.11 tells us that the value of $d$ does not depend on $a^{\prime}$.

$f$ is injective, since $\hat{b}=\tilde{a}^{-1} \cdot\left(a, a^{\prime}, f(\hat{b})\right)$. It is surjective since $\tilde{a}^{-1} \cdot\left(a, a^{\prime}, d\right) \in H$ for every $d \in a \cdot e$.

Corollary 1.14. $H<G$ is a bounded subgroup.

Proof. $H$ is bounded since $a \cdot e$ is bounded for any $a$.

$H$ is clearly closed for inverses. As for products, let $\hat{b}_{i} \in H$ for $i<2$, and let $\tilde{a}=$ $\left(a, a^{\prime}, a^{\prime \prime}\right)$ be quite arbitrary. Then $\tilde{a}_{i}=\hat{b}_{i} \cdot \tilde{a}=\left(a, a_{i}^{\prime}, a^{\prime \prime}\right)$, whereby $\hat{b}_{0} \cdot \hat{b}_{1}^{-1}=\tilde{a}_{0} \cdot \tilde{a}_{1}^{-1} \in$ $H$.

Lemma 1.15. Write $G=G_{0} / R$, where $G_{0}$ is a type-definable set and $R$ an I-graded almost type-definable equivalence relation, whose grading is compatible with the structure of $G$, and similarly $\tilde{S}=\tilde{S}_{0} / R$ (this needs not be the same $R$, strictly speaking, but there is no place for ambiguity).

For just this time we consider real elements rather than $R$-classes.

Set:

$$
\begin{aligned}
& H_{0}=\left\{h \in G_{0}: h_{R} \in H\right\} \\
& H_{1}=\bigcup_{\left(a, a_{i}^{\prime}, a^{\prime \prime}\right)_{i<2} \in \tilde{S}_{0}}\left(a, a_{0}^{\prime}, a^{\prime \prime}\right) \cdot\left(a, a_{1}^{\prime}, a^{\prime \prime}\right)^{-1}
\end{aligned}
$$

Then $H_{1} \subseteq H_{0} \subseteq G_{0}, H_{1}$ is type-definable, and $H_{0}=\bigcup_{i \in I} H_{1} / R_{i}$. Moreover, taking $\left(H_{1} / R_{i}\right)$ as a grading of $H$ (in the same way that $\left(R_{i}\right)$ is a grading of $R$ ), then this grading is compatible with the group structure.

Proof. Clear from the construction.

Corollary 1.16. $G / / H$ is a I-gradedly almost hyperdefinable polygroup, poly-isogenous to $G$.

Remark 1.17. If we do not insist on considering $H$ as a subgroup of $G$, the infinite union is not necessary: there is $i \in I$ such that for every $g, h \in H_{1}$ there is $f \in$ $H_{1} \cap(g \cdot h) / R_{i}$, whereby $H_{1} /\left(R \uparrow_{H_{1}}\right)$ is an almost hyperdefinable group, isomorphic to $H$.

Lemma 1.18. For every independent generic $\tilde{a}, \tilde{b} \in \tilde{S}$, we have in $S: \pi(\tilde{a}) \cdot \pi(\tilde{b})=$ $a \cdot b=\pi(\tilde{a} H \tilde{b})$.

Moreover, the above statements holds in the graded sense, meaning that there is $i \in I$ such that if $\tilde{a}$ and $\tilde{b}$ are actual representatives, then $\pi(\tilde{a}) \cdot \pi(\tilde{b}) \subseteq \pi\left(\tilde{a} H_{1} \tilde{b}\right) / R_{i}$ and $\pi\left(\tilde{a} H_{1} \tilde{b}\right) \subseteq(\pi(\tilde{a}) \cdot \pi(\tilde{b})) / R_{i}$. 
Proof. We have $\pi(\tilde{a} \cdot \hat{c})=a$ for every $\hat{c} \in H$, so: $\pi(\tilde{a} H \tilde{b}) \subseteq \bigcup_{\hat{c} \in H} \pi(\tilde{a} \cdot \hat{c}) \cdot \pi(\tilde{b})=a \cdot b$. For the other inclusion, let $c \in a \cdot b$. Let $b_{1}^{\prime}=a^{\prime \prime}-1 \cdot c \stackrel{a}{\cap} e^{-1} \cdot b$, so $c=a \cdot b \stackrel{e}{\cap} a^{\prime \prime} \cdot b_{1}^{\prime}$. Therefore, setting $\tilde{b}_{1}=\left(b, b_{1}^{\prime}, b^{\prime \prime}\right)$, we get:

$$
\begin{aligned}
c & =\pi\left(\tilde{a} \cdot \tilde{b}_{1}\right)=\pi\left(\tilde{a} \cdot\left(\tilde{b}_{1} \cdot \tilde{b}^{-1}\right) \cdot \tilde{b}\right) \\
& \in \pi(\tilde{a} H \tilde{b})
\end{aligned}
$$

The graded version is proved precisely the same, with some additional bookkeeping that is easier to verify by oneself than to read (and would be very cumbersome to write).

1.3. The blow-up of a polygroup. We assume now that $P$ is a coreless gradedly almost hyperdefinable polygroup, and $S$ its set of generic elements (so it is a coreless polygroup chunk). The rest is as above.

We let $\hat{\pi}: G \rightarrow P$ be the unique extension of the blow-up map $\pi: \tilde{S} \rightarrow S$, by [BTW01, Theorem 4.4]. We recall that for $g \in G$ and $h_{0}, h_{1} \in G$ generic and independent over $g: \pi\left(h_{0}\right) \cdot \pi\left(h_{0}^{-1} \cdot g\right) \cap \pi\left(h_{1}\right) \cdot \pi\left(h_{1}^{-1} \cdot g\right)=\left(\pi\left(h_{0}\right) \cdot \pi\left(h_{0}^{-1} \cdot g\right)\right) \cap \operatorname{bdd}(g)=$ $\{\hat{\pi}(g)\}$.

Lemma 1.19. $H=\operatorname{ker} \hat{\pi}$.

Proof. Let $\hat{b} \in H$, say $\hat{b}=\tilde{a}_{0} \cdot \tilde{a}_{1}^{-1}$. Then $\hat{b} \downarrow \tilde{a}_{0}$ and $e \in \pi\left(\tilde{a}_{0}\right) \cdot \pi\left(\tilde{a}_{1}^{-1}\right) \cap \operatorname{dcl}(\hat{b})$, so $\hat{\pi}(\hat{b})=e$.

Conversely, assume that $\hat{\pi}(g)=e$ for some $g \in G$. Choose $\tilde{a}_{0} \downarrow g$, and set $\tilde{a}_{1}=g^{-1} \cdot \tilde{a}_{0}$. Then $\hat{\pi}(g)=e \in \pi\left(\tilde{a}_{0}\right) \cdot \pi\left(\tilde{a}_{1}^{-1}\right)$, so $\tilde{a}_{0}$ and $\tilde{a}_{1}$ have the same axis: $\tilde{a}_{i}=\left(a, a_{i}^{\prime}, a_{i}^{\prime \prime}\right)$. Choose $\left(b, b^{\prime}, b^{\prime \prime}\right)=\tilde{b} \downarrow g \tilde{a}_{0} \tilde{a}_{1}$ and set $\tilde{c}_{i}=\tilde{a}_{i} \cdot \tilde{b}$. Then $g=\tilde{c}_{0} \cdot \tilde{c}_{1}^{-1}$ and $\tilde{c}_{i} \downarrow g$, and by the same argument: $\tilde{c}_{i}=\left(c, c_{i}^{\prime}, c_{i}^{\prime \prime}\right)$. But then $c_{0}^{\prime \prime}=a \cdot b^{\prime \prime} \stackrel{b}{\cap} c \cdot e=c_{1}^{\prime \prime}$, so $g=\tilde{c}_{0} \cdot \tilde{c}_{1}^{-1} \in H$.

And Lemma 1.18 generalises to:

Lemma 1.20. For every $g, h \in G$ we have in $P: \hat{\pi}(g) \cdot \hat{\pi}(h)=\hat{\pi}(g H h)$. As in Lemma 1.18, the graded version holds as well.

Proof. One inclusion is easy: $\hat{\pi}(g H h) \subseteq \hat{\pi}(g) \cdot \hat{\pi}(H) \cdot \hat{\pi}(h)=\hat{\pi}(g) \cdot \hat{\pi}(h)$.

We have $g, h \in G$ and $c \in \hat{\pi}(g) \cdot \hat{\pi}(h)$, and we try to reduce to the case treated in Lemma 1.18. Choose two independent generics $f, f^{\prime}$ over $g h$. Then $c \in \hat{\pi}\left(g \cdot f^{-1}\right)$. $\hat{\pi}(f) \cdot \hat{\pi}\left(f^{\prime}\right) \cdot \hat{\pi}\left(f^{\prime-1} \cdot h\right)$. Applying associativity we obtain $\left[\hat{\pi}\left(f \cdot g^{-1}\right) \cdot c \cdot \hat{\pi}\left(h^{-1} \cdot f^{\prime}\right)\right] \cap[\hat{\pi}(f)$. $\left.\hat{\pi}\left(f^{\prime}\right)\right] \neq \varnothing$. By Lemma 1.18 there is $\hat{a} \in H$ such that $\hat{\pi}\left(f \hat{a} f^{\prime}\right) \in \hat{\pi}\left(f \cdot g^{-1}\right) \cdot c \cdot \hat{\pi}\left(h^{-1} \cdot f^{\prime}\right)$. Now let $f$ vary as $f_{i}$, which we may take to be all independent over $g h$, and for each pair we get $\hat{a}_{i} \in H$ such that $\hat{\pi}\left(f_{i} \cdot \hat{a}_{i} \cdot f^{\prime}\right) \in \hat{\pi}\left(f_{i} \cdot g^{-1}\right) \cdot c \cdot \hat{\pi}\left(h^{-1} \cdot f^{\prime}\right)$. Let $d_{i} \in c \cdot \hat{\pi}\left(h^{-1} \cdot f^{\prime}\right)$ be such that $\hat{\pi}\left(f_{i} \cdot \hat{a}_{i} \cdot f^{\prime}\right) \in \hat{\pi}\left(f_{i} \cdot g^{-1}\right) \cdot d_{i}$. Then there are boundedly many possibilities for $d_{i}$ and $\hat{a}_{i}$, so there exist two values of $i$ (say 0 and 1) for which they are the same. Note $\hat{a}=\hat{a}_{0}=\hat{a}_{1}$ and $d=d_{0}=d_{1}$. Then we have for $i \in 2$ : $d \in \hat{\pi}\left(g \cdot f_{i}^{-1}\right) \cdot \hat{\pi}\left(f_{i} \cdot \hat{a} \cdot f^{\prime}\right)$. As $f_{0}$ and $f_{1}$ were chosen independent from everything, we get: $d=\hat{\pi}\left(g \cdot f_{i}^{-1} \cdot f_{i} \cdot \hat{a} \cdot f^{\prime}\right)=\hat{\pi}\left(g \cdot \hat{a} \cdot f^{\prime}\right)$. Now let $f^{\prime}$ vary, and proceed similarly on the right to find $\hat{a} \in H$ such that $c=\hat{\pi}(g \cdot \hat{a} \cdot h)$.

For the graded version, work with representatives: since $R$ is almost type-definable, we can actually find two values of $i$ (say, 0 and 1 ) such that $\hat{a}_{0} R_{0} \hat{a}_{1}$ and $d_{0} R_{0} d_{1}$. 
We obtain:

Theorem 1.21. Let $P$ be a coreless gradedly almost hyperdefinable polygroup, $S$ the set of its generic elements, and $\tilde{S}, G, H$ as above. Then $P \simeq G / / H$.

Proof. Let $\sigma: G / / H \rightarrow P$ be induced by $\hat{\pi}$.

We show first that it is surjective. For an element $a \in P$, let $b$ and $c$ be independent generics of $P$ over $a$. We can write $a \in b \cdot b^{\prime} \cap c \cdot c^{\prime}$ for some $b^{\prime}, c^{\prime}$ (which are also generic and independent over $a$ ). By associativity we find $d \in b^{-1} \cdot c \cap b^{\prime} \cdot c^{\prime-1}$, and it is generic because $b \downarrow c$. Choose a blow-up $\tilde{d}$ of $d$ : then there are blow-ups $\tilde{b}, \tilde{c}, \tilde{b}^{\prime}, \tilde{c}^{\prime}$ such that $\tilde{d}=\tilde{b}^{-1} \cdot \tilde{c}=\tilde{b}^{\prime} \cdot \tilde{c}^{\prime}-1$. Write $g=\tilde{b} \cdot \tilde{b^{\prime}}=\tilde{b} \cdot \tilde{d} \cdot \tilde{c}^{\prime}=\tilde{c} \cdot \tilde{c}^{\prime}$. Then $b \downarrow a c \Longrightarrow b \downarrow c c^{\prime} \Longrightarrow \tilde{b} \downarrow g \tilde{c}$ and similarly $\tilde{c} \downarrow g \tilde{b}$ so $\hat{\pi}(g)=a$.

We also know that $\operatorname{ker} \sigma=\left\{\bar{e}_{G}\right\}$, and $\sigma(\bar{g} \cdot \bar{h})=\sigma(\bar{g}) \cdot \sigma(\bar{h})$ (by Lemma 1.20), $\sigma\left(\bar{g}^{-1}\right)=$ $\sigma(\bar{g})^{-1}$. Then:

$$
\begin{aligned}
\sigma(\bar{g})=\sigma(\bar{h}) & \Longrightarrow e_{P} \in \sigma(\bar{g}) \cdot \sigma(\bar{h})^{-1}=\sigma\left(\bar{g} \cdot \bar{h}^{-1}\right) \\
& \Longrightarrow \bar{e}_{G} \in \bar{a} \cdot \bar{b}^{-1} \\
& \Longrightarrow \bar{a}=\bar{b}
\end{aligned}
$$

And $\sigma$ is injective.

Remark 1.22. If $P$ is not coreless, then $P / N \simeq G / / H$, where $N \triangleleft P$ is the core of $P$, and we recall that this is a bounded normal sub-polygroup.

Remark 1.23. We did not find in the (poly)algebraic literature any reference to the question of what polygroups can be written as double-coset spaces (although double-coset spaces are often mentioned as an important example of polygroups).

In [Com84], Comer orders (linearly) by weak inclusion certain similar properties, the strongest of which, $Q^{2}$, being weaker than double-coset space. He also asks whether any of the inclusions is strict, but gives no consideration to the inclusion of double-coset spaces in $Q^{2}$ : if this is due to an evident counter-example, then we are not aware of it.

Theorem 1.24. Let $S$ be a coreless gradedly almost hyperdefinable polygroup chunk, and $\tilde{S}, G, H$ as above. Then $G / / H$ is coreless, and $\operatorname{gen}(G / / H) \simeq S$, where $\operatorname{gen}(G / / H)$ denotes the set of the generic elements.

Moreover, $G / / H$ is unique (up to a unique isomorphism) with these properties.

Proof. By Proposition 1.13, the set $H \tilde{a} H$ is precisely the set of all blow-ups of $a$, so $S$ and $\operatorname{gen}(G / / H)$ are in natural bijection. This bijection is an isomorphism of generic chunks by Lemma 1.18 .

Let $P$ be any coreless polygroup such that $S \simeq \operatorname{gen}(P)$. Then by the previous theorem $P \simeq G / / H$, whence the uniqueness. In particular, this holds for the core-reduct of $G / / H$, so $G / / H$ is coreless.

This may be viewed as a polygroup chunk theorem, although we needed to pass through the group chunk theorem in order to prove it. 


\section{The $\varnothing$-Definable CATEgory OF BLOW-UP GROUPS}

One apparent disadvantage of the blow-up construction is the introduction of the parameter $e$ : as we start with a $\varnothing$-definable polygroup chunk, we would like to end up with a $\varnothing$-definable object. Even in the stable case one may need to increase the set of parameters in order to pass from an algebraic quadrangle where everything is algebraic to one where everything is actually defined, so this is not surprising. On the other hand, as here we do it at a later stage, we may understand better what is going on, and what can be recovered that is $\varnothing$-definable.

In his $\mathrm{PhD}$ thesis [Tom01], Tomašić uses tools similar to those we describe here in a manner opposite to ours: by fixing a big set of independent $e_{i}$ and compatible $\varphi_{e_{i}, e_{j}, f_{i j}} \in I\left(e_{i}, e_{j}\right)$ (see below), he obtains a blown-up group chunk with a universal property with respect to these parameters.

2.1. The category of arbitrary choices. Here $S$ is still a coreless polygroup chunk. However, we will now let $e \in S_{0}$ vary, so we obtain blow-ups $\tilde{S}_{e}$ and groups $G_{e}$. Note that the definitions of $G_{e}$ in terms of $e$ are uniform.

Had it been true that $G_{e}$ and $G_{e^{\prime}}$ were canonically isomorphic for every e, $e^{\prime} \in S$, then we would have obtained a $\varnothing$-definable $G$ canonically isomorphic to any $G_{e}$. Unfortunately, although they are isomorphic, generally there is no one canonical isomorphism that stands out. Instead, we can isolate a small (that is, bounded) set of isomorphisms which is canonical.

Construction 2.1. Assume we have $e \downarrow e^{\prime}$, and fix some $f \in e^{-1} \cdot e^{\prime}$. Consider now $a \downarrow e e^{\prime}$, and $\tilde{a}=\left(a, a^{\prime}, a^{\prime \prime}\right) \in \tilde{S}_{e}$, and set $b^{\prime}=e^{\prime-1} \cdot a \stackrel{e}{\cap} f^{-1} \cdot a^{\prime}$ and $b^{\prime \prime}=a \cdot e^{\prime} \stackrel{e}{\cap} a^{\prime \prime} \cdot f$. Then sending $\left(a, a^{\prime}, a^{\prime \prime}\right)$ to $\left(a, b^{\prime}, b^{\prime \prime}\right)$ gives us a map from a generic enough part of $\tilde{S}_{e}$ to $\tilde{S}_{e^{\prime}}$, which can be seen to preserve products. This induces a definable homomorphism $\varphi_{e, e^{\prime}, f}: G_{e} \rightarrow G_{e^{\prime}}$. One also sees that $\varphi_{e, e^{\prime}, f}^{-1}=\varphi_{e^{\prime}, e, f^{-1}}$, so this is an isomorphism. Write $I\left(e, e^{\prime}\right)=\left\{\varphi_{e, e^{\prime}, f}: f \in e^{-1} \cdot e^{\prime}\right\}$.

We recall [BTW01, Corollary 3.3]:

Fact 2.2. Let $a_{1}, a_{2}, b_{1}, b_{2}, c_{1}, c_{2}, d_{1}, d_{2} \in S$ satisfy:

1. $\left\{a_{1}, a_{2}, d_{1}, d_{2}\right\}$ are independent.

2. $\left.d_{1} \in a_{1}^{-1} \cdot b_{1} \cap a_{2} \cdot b_{2}^{-1}\right)$ and $d_{2} \in a_{1}^{-1} \cdot c_{1} \cap a_{2} \cdot c_{2}^{-1}$.

3. $b_{1}^{-1} \cdot c_{1} \cap d_{1}^{-1} \cdot d_{2} \cap b_{2} \cdot c_{2}^{-1} \neq \varnothing$.

Then $a_{1} \cdot a_{2} \stackrel{d_{1}}{\cap} b_{1} \cdot b_{2}=a_{1} \cdot a_{2} \stackrel{d_{2}}{\cap} c_{1} \cdot c_{2}$ :

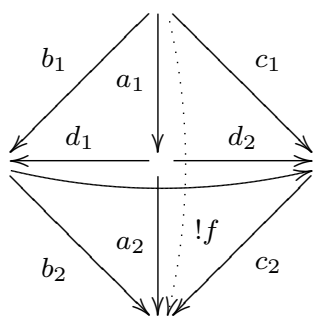


Lemma 2.3. $\quad$ 1. Let $e \downarrow e^{\prime}$. Then the map $f \mapsto \varphi_{e, e^{\prime}, f}$ is bijection between $e^{-1} \cdot e^{\prime}$ and $I\left(e, e^{\prime}\right)$. In fact, if $\varphi_{e, e^{\prime}, f}$ and $\varphi_{e, e^{\prime}, f^{\prime}}$ coincide on an element of $G_{e}$ generic over $e^{\prime}$, then $f=f^{\prime}$.

2. Assume that $\left\{e, e^{\prime}, e^{\prime \prime}\right\}$ are independent, $f \in e^{-1} \cdot e^{\prime}, f^{\prime} \in e^{-1} \cdot e^{\prime \prime}$ are given, and set $f^{\prime \prime}=e^{-1} \cdot e^{\prime \prime} \stackrel{e^{\prime}}{\cap} f \cdot f^{\prime}$. Then $\varphi_{e, e^{\prime \prime}, f^{\prime \prime}}=\varphi_{e^{\prime}, e^{\prime \prime}, f^{\prime}} \circ \varphi_{e, e^{\prime}, f}$.

3. For every independent $\left\{e, e^{\prime}, e^{\prime \prime}\right\}$ and $\varphi \in I\left(e, e^{\prime}\right), \varphi^{\prime} \in I\left(e^{\prime}, e^{\prime \prime}\right)$ we have $I\left(e, e^{\prime \prime}\right)=$ $I\left(e^{\prime}, e^{\prime \prime}\right) \circ \varphi=\varphi^{\prime} \circ I\left(e, e^{\prime}\right)$.

4. Assume that $e^{\prime \prime} \downarrow e e^{\prime}$ (but $e, e^{\prime}$ are not necessarily independent). Then for every $\varphi \in I\left(e, e^{\prime \prime}\right)$ and $\varphi^{\prime} \in I\left(e^{\prime \prime}, e^{\prime}\right)$ we have $I\left(e^{\prime \prime}, e^{\prime}\right) \circ \varphi=\varphi^{\prime} \circ I\left(e, e^{\prime \prime}\right)=I\left(e^{\prime \prime}, e^{\prime}\right) \circ$ $I\left(e, e^{\prime \prime}\right)$.

Moreover, this set does not depend on $e^{\prime \prime}$.

Proof. 1. By definition it is surjective. Let $\tilde{a} \in \tilde{S}_{e}$ be generic over $e^{\prime}$ (that is, $\left.a \downarrow e e^{\prime}\right)$, and assume that $\varphi_{e, e^{\prime}, f}(\tilde{a})=\varphi_{e, e^{\prime}, f^{\prime}}(\tilde{a})=\left(a, b^{\prime}, b^{\prime \prime}\right)$. Then we have both $f=e^{-1} \cdot e^{\prime} \stackrel{a}{\cap} a^{\prime} \cdot b^{\prime-1}=f^{\prime}$ and $f=a^{\prime \prime}-1 \cdot b^{\prime \prime} \stackrel{a}{\cap} e^{-1} \cdot e^{\prime}=f^{\prime}$ (so in fact, it suffices that $\varphi_{e, e^{\prime}, f}(\tilde{a})$ and $\varphi_{e, e^{\prime}, f^{\prime}}(\tilde{a})$ have the same extension on one side).

2. It suffices to prove the statement for $\tilde{a} \in \tilde{S}_{e}$ such that $a \downarrow e e^{\prime} e^{\prime \prime}$. Write $\left(a, b^{\prime}, b^{\prime \prime}\right)=\varphi_{e, e^{\prime}, f}(\tilde{a}),\left(a, c^{\prime}, c^{\prime \prime}\right)=\varphi_{e^{\prime}, e^{\prime \prime}, f^{\prime}} \circ \varphi_{e, e^{\prime}, f}(\tilde{a})$ and $\left(a, d^{\prime}, d^{\prime \prime}\right)=\varphi_{e, e^{\prime \prime}, f^{\prime \prime}}(\tilde{a})$. Then we have $b^{\prime \prime}=a \cdot e^{\prime} \stackrel{e}{\cap} a^{\prime \prime} \cdot f, c^{\prime \prime}=a \cdot e^{\prime \prime} \stackrel{e^{\prime}}{\cap} b^{\prime \prime} \cdot f^{\prime}$ and $d^{\prime \prime}=a \cdot e^{\prime \prime} \stackrel{e}{\cap} a^{\prime \prime} \cdot f^{\prime \prime}$. Then the assumptions of Fact 2.2 hold, whereby $c^{\prime \prime}=d^{\prime \prime}$ :

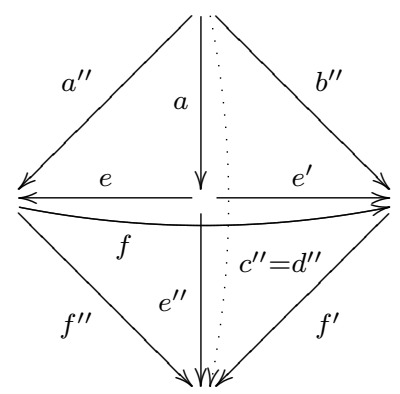

One proves $c^{\prime}=d^{\prime}$ similarly.

3. For every $\varphi^{\prime \prime} \in I\left(e, e^{\prime \prime}\right)$ we have $\varphi^{\prime \prime} \circ \varphi^{-1} \in I\left(e^{\prime}, e^{\prime \prime}\right), \varphi^{\prime-1} \circ \varphi^{\prime \prime} \in I\left(e, e^{\prime}\right)$.

4. It suffices to prove that for every $e^{\prime \prime} \downarrow e e^{\prime}, e^{\prime \prime \prime} \downarrow e e^{\prime} e^{\prime \prime}$, and for every $\varphi \in I\left(e, e^{\prime \prime}\right)$, $\varphi^{\prime} \in I\left(e^{\prime \prime \prime}, e^{\prime}\right)$ we have $I\left(e^{\prime \prime}, e^{\prime}\right) \circ \varphi=\varphi^{\prime} \circ I\left(e, e^{\prime \prime \prime}\right)$. This is equivalent to showing that $\varphi^{\prime-1} \circ I\left(e^{\prime \prime}, e^{\prime}\right)=I\left(e, e^{\prime \prime \prime}\right) \circ \varphi^{-1}$, but we know that both sets are equal to $I\left(e^{\prime \prime}, e^{\prime \prime \prime}\right)$.

And we obtain:

Proposition 2.4. For every $e, e^{\prime} \in S$ set $I\left(e, e^{\prime}\right)=I\left(e^{\prime \prime}, e^{\prime}\right) \circ I\left(e, e^{\prime \prime}\right)$ for some $e^{\prime \prime} \downarrow e e^{\prime}$. Then this extends Construction 2.1 and does not depend on $e^{\prime \prime}$.

Moreover, let $\mathcal{I}$ be the category of groups given by:

$$
\begin{gathered}
\operatorname{obj}(\mathcal{I})=\left\{G_{e}: e \in S\right\} \\
\operatorname{Hom}_{\mathcal{I}}\left(G_{e}, G_{e^{\prime}}\right)=I\left(e, e^{\prime}\right)
\end{gathered}
$$


Then $\mathcal{I}$ is the unique category with these objects such that * holds for $e \downarrow e^{\prime}$.

Proof. This is just a re-statement of Lemma 2.3.

This can be more loosely restated by saying that $\mathcal{I}$ is the $\varnothing$-invariant structure that describes our inability to define $G$ over $\varnothing$. In order to say that $\mathcal{I}$ is rather $\varnothing$-definable, we need:

Definition 2.5. Let $\mathcal{C}=\mathcal{C}_{0} / R$ be an I-gradedly almost hyperdefinable set, and obj a hyperdefinable (and not almost hyperdefinable) set. Let $\overline{\mathrm{o}}: \mathcal{C}^{2} \rightarrow \mathcal{C}$ be a partial gradedly definable map, and let $\overline{i d}:$ obj $\rightarrow \mathcal{C}$, and $\bar{l}, \bar{r}: \mathcal{C} \rightarrow$ obj be gradedly definable maps. For $a, b \in$ obj we write: $\operatorname{Hom}_{\mathcal{C}}(a, b)_{0}=\left\{f \in \mathcal{C}_{0}: l(f)=a, r(f)=b\right\}, \operatorname{Hom}_{\mathcal{C}}(a, b)=$ $\operatorname{Hom}_{\mathcal{C}}(a, b)_{0} / R$. Note that since obj is hyperdefinable, $l$ and $r$ just take one value, even before passing to $\bar{l}$ and $\bar{r}$; in particular, if $f \in \operatorname{Hom}_{\mathcal{C}}(a, b)_{0}$ then $f / R \subseteq \operatorname{Hom}_{\mathcal{C}}(a, b)_{0}$. We now require that the following axioms be satisfied in the graded sense:

1. $r(f)=l(g) \leftrightarrow l(f \circ g)=l(f) \leftrightarrow r(f \circ g)=r(f)$

2. $r(f)=l(g) \wedge r(g)=l(h) \rightarrow(f \circ g) \circ h=f \circ(g \circ h)$

3. $r(i d(x))=l(i d(x))=x$

4. $f \circ i d(r(f))=i d(l(f)) \circ f=f$

If all this holds, then $\mathcal{C}$ is an $I$-gradedly almost hyperdefinable category.

Remark 2.6. The reason for which obj is assumed to be hyperdefinable rather than almost hyperdefinable, as one might have expected, is that we never allow almost hyperimaginaries as parameters, and yet $\operatorname{Hom}_{\mathcal{C}}(a, b)$ is defined over ab.

Ordinarily, this does not pose any significant limitations on what we can do. However, if we really must have obj almost hyperdefinable, say obj $=o b j_{0} / R^{\prime}$, the obstacle can be overcome by defining an identity in $\operatorname{Hom}_{\mathcal{C}}(a, b)$ whenever a $R^{\prime} b$ :

Replace everywhere in Definition 2.5 obj with objo, and say that for every $i \in I$ we have $\overline{i d}_{i}: R_{i}^{\prime} \rightarrow \mathcal{C}$ such that a $R_{i} b \Longrightarrow i d_{i}(a, b) \in \operatorname{Hom}_{\mathcal{C}}(a, b)$, if $j \geq i$ then $i d_{i}(a, b)=i d_{j}(a, b)$, and the axiom for the identity is modified accordingly (in particular, now we need to say that the composition of two identities is an identity).

Dividing by (composition with) these identities, we obtain $\operatorname{Hom}_{\mathcal{C}}(a, b)$ for $a, b \in$ obj.

Proposition 2.7. $\mathcal{I}$ is an almost hyperdefinable category.

Proof. First, for $e_{R} \downarrow e_{R}^{\prime}$, define $I_{0}\left(e_{R}, e_{R}^{\prime}\right)=e^{-1} \cdot e^{\prime}$. This is a type-definable set (and the set $\left\{\left(e, e^{\prime}\right) \in S_{0}^{2}: e_{R} \downarrow e_{R}^{\prime}\right\}$ is also type-definable). For independent $\left\{e_{R}, e_{R}^{\prime}, e_{R}^{\prime \prime}\right\}$, and $f \in I_{0}\left(e, e^{\prime}\right), f^{\prime} \in I_{0}\left(e^{\prime}, e^{\prime \prime}\right)$, define $f \circ_{0} f^{\prime}=e^{-1} \cdot e^{\prime \prime} \cap f \cdot f^{\prime}$. Since we deal with real elements, $f \circ_{0} f^{\prime}$ is a type-definable set, and $\left(f \circ_{0} f^{\prime}\right)_{R}=e_{R}^{-1} \cdot e_{R}^{\prime \prime} e_{R}^{\prime} f_{R} \cdot f_{R}^{\prime}$ is a singleton.

Now set for any $e, e^{\prime} \in S_{0}$ :

$$
\begin{aligned}
\mathcal{I}_{0} & =\left\{\left(e, e^{\prime}, e^{\prime \prime}, \varphi, \varphi^{\prime}\right): e_{R}^{\prime \prime} \downarrow e e^{\prime}, \varphi \in I_{0}\left(e, e^{\prime \prime}\right), \varphi^{\prime} \in I_{0}\left(e^{\prime \prime}, e^{\prime}\right)\right\} \\
\mathcal{I}_{0}\left(e, e^{\prime}\right) & =\left\{\left(f, f^{\prime}, f^{\prime \prime}, \varphi, \varphi^{\prime}\right) \in \mathcal{I}_{0}: f=e, f^{\prime}=e^{\prime}\right\} \\
\left(e, e^{\prime}, f, \varphi, \varphi^{\prime}\right) & \circ_{1}\left(e^{\prime}, e^{\prime \prime}, f^{\prime}, \psi, \psi^{\prime}\right)= \\
= & \left\{\left(e, e^{\prime \prime}, f^{\prime \prime}, \chi, \chi^{\prime}\right) \in \mathcal{I}_{0}: f_{R}^{\prime \prime} \downarrow e e^{\prime} e^{\prime \prime} f f^{\prime} \wedge\right. \\
& \left.\chi^{\prime} \in\left(\psi^{\prime} \circ\left(\psi \circ\left(\varphi^{\prime} \circ\left(\varphi \circ \chi^{-1}\right)\right)\right)\right)\right\}
\end{aligned}
$$


Define $\left(e, e^{\prime}, e^{\prime \prime}, \varphi, \varphi^{\prime}\right) \equiv_{i}\left(f, f^{\prime}, f^{\prime \prime}, \psi, \psi^{\prime}\right)$ on $\mathcal{I}_{0}$ as:

$$
\begin{aligned}
\exists e^{\prime \prime \prime}, \tau e & =f \wedge e^{\prime}=f^{\prime} \\
& \wedge e_{R}^{\prime \prime \prime} \downarrow e e^{\prime} e^{\prime \prime} f^{\prime \prime} \wedge \tau \in I_{0}\left(e^{\prime \prime \prime}, e\right) \\
& \wedge \varphi^{\prime} \circ_{0}\left(\varphi \circ_{0} \tau\right) R_{i} \psi^{\prime} \circ_{0}\left(\psi \circ_{0} \tau\right)
\end{aligned}
$$

Then $\equiv$ is an $I$-graded almost type-definable equivalence relation on $\mathcal{I}_{0}$, and $I\left(e_{R}, e_{R}^{\prime}\right)$ is in natural bijection with $\mathcal{I}_{0}\left(e, e^{\prime}\right) / \equiv$. In particular, if $e R f$ and $e^{\prime} R f^{\prime}$ then $\mathcal{I}_{0}\left(e, e^{\prime}\right) / \equiv \simeq \mathcal{I}_{0}\left(f, f^{\prime}\right) / \equiv$ in a gradedly unique canonical manner; still, it is convenient to keep the distinction between $\mathcal{I}_{0}\left(e, e^{\prime}\right) / \equiv$ and $\mathcal{I}_{0}\left(f, f^{\prime}\right) / \equiv$, since this way the base sets $\mathcal{I}_{0}\left(e, e^{\prime}\right)$ and $\mathcal{I}_{0}\left(f, f^{\prime}\right)$ are type-definable. Composition $\bar{o}_{1}: \mathcal{I}_{0}\left(e, e^{\prime}\right) / \equiv \times \mathcal{I}_{0}\left(e^{\prime}, e^{\prime \prime}\right) / \equiv \rightarrow$ $\mathcal{I}_{0}\left(e, e^{\prime \prime}\right) / \equiv$ is a graded map (that is, compatible with the grading of $\equiv$ ).

It should now be evident to define $r, l$ and $i d$ so as to make $\mathcal{I}=\mathcal{I}_{0} / \equiv$ an $I$-gradedly almost hyperdefinable category, with $o b j=S_{0}$.

Remark 2.8. In fact, the action of $\mathcal{I}\left(e, e^{\prime}\right)$ from $G_{e}$ to $G_{e^{\prime}}$ is also almost hyperdefinable, so we might say that $\mathcal{I}$ is an almost hyperdefinable concrete category.

One easily verifies that the approach suggested in Remark 2.6 can be applied in this case make the set of objects $S$ rather than $S_{0}$.

2.2. The subgroup $H$ revisited. For $e \downarrow e^{\prime}$, the interpretation of $I\left(e, e^{\prime}\right)$ is given by Proposition 2.4: a small set of isomorphisms between $G_{e}$ and $G_{e^{\prime}}$, constructed rather naturally, of which none is distinguished above the others (as no element of the set $e^{-1} \cdot e^{\prime}$ is distinguished above its peers). This also determines $I\left(e, e^{\prime}\right)$ for any $e, e^{\prime}$, and in particular $I(e, e)<\operatorname{Aut}\left(G_{e}\right)$. However, in $I(e, e)$ we have clearly one distinguished element, namely the identity, so the interpretation for independent $e, e^{\prime}$ no longer holds. Instead, it interprets in terms of $H_{e}$ :

Proposition 2.9. There is a gradedly definable isomorphism $i: I(e, e) \simeq H_{e}$ which coincides with the action of $H_{e}$ on $G_{e}$ through internal automorphisms: $\varphi(g)=g^{i(\varphi)}$.

Proof. Fix some $\tilde{f}=\left(f, f^{\prime}, f^{\prime \prime}\right) \in \tilde{S}_{e}$. Then $f^{\prime} \in e^{-1} \cdot f$ and $\varphi_{e, f, f^{\prime}} \in I(e, f)$.

Let $\psi \in I(e, e)$. Then there is a unique $f_{\psi}^{\prime} \in e^{-1} \cdot f$ (that is, a unique $\varphi_{e, f, f_{\psi}^{\prime}} \in I(e, f)$ ) such that $\psi=\varphi_{e, f, f^{\prime}}^{-1} \circ \varphi_{e, f, f_{\psi}^{\prime}}$. We also have $\tilde{f}_{\psi}=\left(f, f_{\psi}^{\prime}, f^{\prime \prime}\right) \in \tilde{S}_{e}$, and we set $i(\psi)=$ $\tilde{f}_{\psi} \cdot \tilde{f}^{-1} \in H_{e}$.

Let $\tilde{a}=\left(a, a^{\prime}, a^{\prime \prime}\right) \in S_{e}$ be generic over $f$, and set:

$$
\begin{array}{ll}
b^{\prime}=f^{-1} \cdot a \stackrel{e}{\cap} f_{\psi}^{\prime-1} \cdot a^{\prime} & b^{\prime \prime}=a \cdot f \stackrel{e}{\cap} a^{\prime \prime} \cdot f_{\psi}^{\prime} \\
c^{\prime}=e^{-1} \cdot a \stackrel{f}{\cap} f^{\prime} \cdot b^{\prime} & c^{\prime \prime}=a \cdot e \stackrel{f}{\cap} b^{\prime \prime} \cdot f^{\prime-1}
\end{array}
$$

Then by Lemma 1.11, and by the definition of $\varphi_{e, f, f_{\psi}^{\prime}}, \varphi_{e, f, f^{\prime}}$ :

$$
\begin{aligned}
\left(a, a^{\prime}, c^{\prime \prime}\right) & =\tilde{a} \cdot i(\psi) \\
\left(a, c^{\prime}, a^{\prime \prime}\right) & =i(\psi)^{-1} \cdot \tilde{a} \\
\psi(\tilde{a})=\left(a, c^{\prime}, c^{\prime \prime}\right) & =\tilde{a}^{i(\psi)}
\end{aligned}
$$


From ${ }^{*}$, and from the fact that elements of $I(e, e)$ act independently on each one-sided extension, we deduce that $i: I(e, e) \rightarrow H_{e}$ is group homomorphism that does not depend of $\tilde{f}$.

As ${ }^{* *}$ holds for every $\tilde{a} \in \tilde{S}_{e}$ generic over $f$, we deduce $\varphi(g)=g^{i(\varphi)}$ for every $g \in G_{e}$. Thus $i$ is injective, and it is surjective since every $f_{\psi}^{\prime} \in e^{-1} \cdot f$ is possible. We conclude: $i: I(e, e) \simeq H_{e}$.

By its construction, $i$ is gradedly definable.

This only describes $I(e, e)$, that is $I(e, f)$ when $e=f$. In order to describe all of $\mathcal{I}$ in similar terms, one has to notice that $\mathcal{I}$ is more than a mere category. We recall:

Definition 2.10. A groupoid (over a set $X$ ) is a category $\mathcal{G}$ where every morphism is invertible (and $X=\operatorname{obj}(\mathcal{G})$ ). It is connected if $\operatorname{Hom}_{\mathcal{G}}(a, b) \neq \varnothing$ for every $a, b \in \operatorname{obj}(\mathcal{G})$.

Notation 2.11. When dealing with a groupoid $\mathcal{G}$, we shall use the notation (and order) $\therefore: \mathcal{G}_{a b} \times \mathcal{G}_{b c} \rightarrow \mathcal{G}_{a c}$ rather than $\circ: \operatorname{Hom}_{\mathcal{G}}(b, c) \times \operatorname{Hom}_{\mathcal{G}}(a, b) \rightarrow \operatorname{Hom}_{\mathcal{G}}(a, c)$.

Example 2.12. A group $G$ is identified with the groupoid $\mathcal{G}$ over the singleton $\{*\}$, where $\mathcal{G}_{* *}=G$.

Example 2.13. Let $X$ be a topological space. Let obj $\left(\boldsymbol{\pi}_{1}(X)\right)=X$, and let $\operatorname{Hom}_{\pi_{1}(X)}(x, y)$ be the set of homotopy classes of paths from $x$ to $y$. Define composition in the obvious manner. Then $\boldsymbol{\pi}_{1}(X)$ is the fundamental groupoid of $X$, and $\operatorname{Hom}_{\pi_{1}(X)}(x, x)=\pi_{1}(X, x)$ for every $x \in X$. It is connected if and only if $X$ is pathconnected.

The fundamental groupoid of a space allows us to turn around the fact that, although $\pi_{1}(X, x)$ is isomorphic to $\pi_{1}(X, y)$ for every $x, y \in X$, there is no natural choice of such an isomorphism.

The reader who appreciates geometric examples is advised that this example is analogous to the situation treated in this paper. In particular, $S$ (or $S_{0}$ ) is the analogue of the base space $X, \mathcal{I}$ is the analogue of $\boldsymbol{\pi}_{1}(X)$, the groups $G_{e}$ form the analogue of a bundle over $X$ (that is, over $S_{0}$ ), and a map $\varphi \in I\left(e, e^{\prime}\right)$ acts as the transport of an element of the bundle along a path.

And of course:

Example 2.14. I is an almost type-definable groupoid (and the inversion map is gradedly definable).

Since what follows is mostly new presentations of previous constructions and results, we skip the details. Let us just say that the theory of stratified local ranks, generic elements, and generic chunks, give in [BTW01] for almost hyperdefinable groups, generalises fully to almost hyperdefinable groupoids. (One just has to remember that when working in $\mathcal{G}_{a b}$, one works over the parameters $a, b$ : in particular, the definition of a generic element $g \in \mathcal{G}_{a b}$ is given in terms of independence over $a b$, etc., and one has to pay attention since $a, b$ may vary as $g$ does.)

The blow-up of a coreless polygroup chunk $S=S_{0} / R$ fits naturally in the context of groupoids. For $e, f \in S_{0}$, define:

$$
\begin{aligned}
\tilde{\mathcal{S}}_{e f, 0} & =\left\{\left(a, a^{\prime}, a^{\prime \prime}\right) \in S_{0}^{3}: a \downarrow e, f \wedge a^{\prime} \in e^{-1} \cdot a \wedge a^{\prime \prime} \in a \cdot f\right\} \\
\tilde{\mathcal{S}}_{\text {ef }} & =\tilde{\mathcal{S}}_{\text {ef }, 0} / R
\end{aligned}
$$


This extends previous definitions, since $\tilde{S}_{e}=\tilde{\mathcal{S}}_{e e}$ for every $e \in S_{0}$. Moreover, if $\tilde{a} \in \tilde{\mathcal{S}}_{e e^{\prime}}, \tilde{b} \in \tilde{\mathcal{S}}_{e^{\prime} e^{\prime \prime}}$, and $\tilde{a} \downarrow e^{\prime \prime} \wedge e \downarrow \tilde{b} \wedge \tilde{a} \downarrow_{e e^{\prime} e^{\prime \prime}} \tilde{b}$ (which is a very complicated way to say that $\left\{a, b, e e^{\prime} e^{\prime \prime}\right\}$ is an independent triplet), then we can define $\tilde{c}=\tilde{a} \cdot \tilde{b} \in \tilde{\mathcal{S}}_{e e^{\prime \prime}}$ just as we did in the case when $e=e^{\prime}=e^{\prime \prime}$. We obtain a generic groupoid chunk $\tilde{\mathcal{S}}$ over $S_{0}$, whence a $\varnothing$-definable groupoid $\mathcal{G}$, where $\mathcal{G}_{e e}=G_{e}$ for every $e \in S_{0}$.

The analogue of $H$ is defined as:

$$
\mathcal{H}_{e_{0} e_{1}}=\left\{\tilde{a}_{0} \cdot \tilde{a}_{1}^{-1}: e^{\prime} \in S, a \downarrow e_{0} e_{1} e^{\prime}, \tilde{a}_{i}=\left(a, a_{i}^{\prime}, a^{\prime \prime}\right) \in \tilde{\mathcal{S}}_{e_{i} e^{\prime}}\right\} \subseteq \mathcal{G}_{e_{0} e_{1}}
$$

All properties proved for $H$ in the first section can be proved just as well for $\mathcal{H}$. In particular, $\mathcal{H}<\mathcal{G}$ is a bounded sub-groupoid (that is, every $\mathcal{H}_{e e^{\prime}}$ is bounded). We can now express $\mathcal{I}$ in terms of $\mathcal{H}$ :

Proposition 2.15. There is a gradedly definable isomorphism $i: \mathcal{I} \simeq \mathcal{H}$ (we identify the object $e \in \operatorname{obj}(\mathcal{H})=\operatorname{obj}(\mathcal{G})$ with $G_{e} \in \operatorname{obj}(\mathcal{I})$ ), such that if $g \in \mathcal{G}_{e e}=G_{e}$ and $\varphi \in I(e, f)$ then $\varphi(g)=g^{i(\varphi)}\left(\right.$ since $i(\varphi) \in \mathcal{H}_{\text {ef }}$ we have $\left.g^{i(\varphi)} \in G_{f}\right)$.

Proof. Same as Proposition 2.9.

Remark 2.16. Let $\varphi \in I(e, f)$ and $\hat{a}=i(\varphi) \in \mathcal{H}_{e f}$. Then $\varphi$ can only act on $G_{e}=\mathcal{G}_{e e}$, but $\hat{a}$ can act on any element $g \in \mathcal{G}_{\cdot e}=\bigcup_{e^{\prime} \in S_{0}} \mathcal{G}_{e^{\prime} e}$ by $g \mapsto g \hat{a}$, and on any element of $\mathcal{G}_{e}$. by $g \mapsto \hat{a}^{-1} g$.

If $e \downarrow f$, then natural variants of Construction 2.1 would give one-sided analogues of $\varphi$ acting one the right $\mathcal{G}_{\cdot e} \rightarrow \mathcal{G}_{\text {.f }}$ and on the left $\mathcal{G}_{e} \rightarrow \mathcal{G}_{f}$, and these would coincide with multiplication by $\hat{a}\left(\hat{a}^{-1}\right)$ on the right (left), respectively.

2.3. Invariance of the polygroup. $\mathcal{I}($ or $\mathcal{H}$ ) describe the arbitrary choices involved in the blow-up: one should expect them to have no effect on the original polygroup (chunk). This is rather clear from the construction, but let us state it formally:

Lemma 2.17. For every $e, e^{\prime} \in S$ and $\varphi \in I\left(e, e^{\prime}\right)$ we have $\varphi\left(H_{e}\right)=H_{e^{\prime}}$.

Proof. We may assume that $e \downarrow e^{\prime}$. Then it suffices to notice that if $\tilde{a}_{i}=\left(a, a_{i}^{\prime}, a^{\prime \prime}\right) \in$ $S_{e}$ are independent of $e^{\prime}$ for $i<2$, then $\varphi\left(\tilde{a}_{i}\right)$ are of the form $\left(a, b_{i}^{\prime}, b^{\prime \prime}\right)$, whereby $\varphi\left(\tilde{a}_{0} \cdot \tilde{a}_{1}^{-1}\right) \in H_{e^{\prime}}$.

(This also follows directly from Proposition 2.15.)

Proposition 2.18. $G_{e} / / H_{e}$ does not depend on e, and is $\varnothing$-definable.

Proof. By Lemma 2.17, every $\varphi \in I\left(e, e^{\prime}\right)$ induces an isomorphism $\bar{\varphi}: G_{e} / / H_{e} \rightarrow$ $G_{e^{\prime}} / / H_{e^{\prime}}$. By Proposition 2.9, this $\bar{\varphi}$ is unique (that is, if $\varphi, \varphi^{\prime} \in I\left(e, e^{\prime}\right)$ then $\left.\bar{\varphi}=\bar{\varphi}^{\prime}\right)$. Define then $G / / H$ as $\left\{(e, \bar{g}): e \in S, \bar{g} \in G_{e} / / H_{e}\right\} / \equiv$ where $(e, \bar{g}) \equiv\left(e^{\prime}, \bar{g}^{\prime}\right)$ if there exists $\varphi \in I\left(e, e^{\prime}\right)$ such that $\bar{\varphi}(\bar{g})=\bar{g}^{\prime}$. This can be done gradedly almost hyperdefinably.

Again, in terms of groupoids this has a more elegant presentation: define $\mathcal{G} / / \mathcal{H}=$ $\{\mathcal{H} g \mathcal{H}: g \in \mathcal{G}\}$ (it is understood that products are only taken when they are defined) and $\mathcal{H} g \mathcal{H} \cdot \mathcal{H} h \mathcal{H}=\mathcal{H} g \mathcal{H} h \mathcal{H}=\mathcal{H} g \mathcal{H}_{r(g) l(h)} h \mathcal{H}$. Then for every $e \in S_{0}$ and class $\mathcal{H} g \mathcal{H}$, there is $g^{\prime} \in G_{e}$ such that $\mathcal{H} g \mathcal{H} \cap G_{e}=H_{e} g^{\prime} H_{e}$, by which the polygroup $P=\mathcal{G} / / \mathcal{H}$ is canonically isomorphic to every $G_{e} / / H_{e}$. We prove that $\mathcal{G} / / \mathcal{H}$ is is almost hyperdefinable as we did for $G_{e} / / H_{e}$, but this time it is over $\varnothing$.

Thus the pair $\mathcal{H}<\mathcal{G}$ contains all the other constructions present in this paper. 


\section{REFERENCES}

[BTW01] Itay Ben-Yaacov, Ivan Tomašić, and Frank O. Wagner, Constructing an almosthyperdefinable group, Preprint, 2001.

[Com84] Stephen D. Comer, Polygroups derived from cogroups, Journal of Algebra 89 (1984), 397405.

[Tom01] Ivan Tomašić, Geometric simplicity theory, Ph.D. thesis, University of Edinburgh, 2001.

[Wag00] Frank O. Wagner, Simple theories, Kluwer Academic Publishers, 2000.

Itay Ben-Yaacov, Équipe de Logique Mathématique, UFR de Mathématiques (Case 7012, Université Paris 7, 2 Place Jussieu, 75251 Paris Cedex 05, France 